\title{
Phenotyping of photosynthetic traits in isogenic chlorophyll-deficient wheat mutant lines
}

\author{
Marián BRESTIC ${ }^{1}$ - Marek ZIVCAK ${ }^{1}$ - Oksana SYTAR ${ }^{1}$ - Viliam BAREK ${ }^{1}$ - \\ Pavol HAUPTFOGEL ${ }^{2}$ \\ 1: Slovak University of Agriculture, Nitra, Slovak Republic \\ 2: National Agricultural and Food Centre, Research Institute of Plant Production, Piestany, Slovak Republic
}

Keywords: photosynthesis, chlorophyll, plant phenotyping

\section{Introduction}

Improving of photosynthetic efficiency has been recently identified as one of the key routes to increase the genetic yield potential of the major crops, including wheat (Long et al. 2015). Although long-term selection for high yields entailed improvement of anatomical and biochemical traits of the leaves of modern high-yielding wheat genotypes (Brestic et al. 2018), there is significant underutilized photosynthetic capacity among existing wheat varieties (Driever et al. 2014). Thus, there are still several ways to improve the photosynthetic efficiency in major crops (Zhu et al. 2010).

\section{Plant material and cultivation}

Biological material: bread wheat (Triticum aestivum L.), wild type (cv. Novosibirskaya 67, NS-67), and chlorophyll-less mutant lines ANBW-4A, ANBW-4B, ANK-32A as well as durum wheat (Triticum durum L.), wild type (cv. LD-222), and chlorophyll-less mutant lines ANDW-7A, ANDW-7B, and ANDW-8A. Wheat plants were grown in pots (nine seedlings per pot) with the standard peat substrate. The pots were regularly irrigated and occasionally fertilized using liquid fertilizer with micronutrients. The cultivation and experiment were carried out in a growth chamber with artificial light provided by fluorescent tubes (growing conditions: $10 / 14 \mathrm{~h}$ dark/light at $18 / 23{ }^{\circ} \mathrm{C}$; PAR at leaf level $\sim 200 \mu \mathrm{mol}$ photons $\mathrm{m}^{-2} \mathrm{~s}^{-1}$ ). The photochemical responses at the PSI and PSII level were analyzed by a Dual-PAM-100 (Walz, Germany) with a ChlF unit and P700 dual wavelength $(830 / 875 \mathrm{~nm})$ unit, as described by Klughammer and Schreiber (1994). The photosynthetic rate was measured by the open infra-red gas exchange system (Licor 6400, Licor, USA). The measurements of gas exchange, ChlF and P700 were analyzed from five repeated measurements.

\section{Results and discussion}

Antenna mutants represent a unique tool to study photosynthetic processes. Moreover, thanks to high variability of leaf and physiological traits (chlorophyll content, growth rate, photosynthetic responses) the collection of mutant and parental lines is particularly useful for phenotyping. In our experiments, we examined in vivo high light responses and photosynthetic capacity of chlorophyll b-less isogenic mutant line of spring wheat (Triticum aestivum L.) - ANBW-4A, ANBW-4B, ANK-32A and durum wheat (Triticum durum L.) - ANDW-7A, ANDW-7B, ANDW-8A, ANDW-8B in comparison to parental lines representing the wild type (WT) in different growth phases. The mutants differed significantly in chlorophyll content and growth rate. Whereas in initial growth phases, the chlorina-phenotype effect was dominant in all mutant lines, in later growth phases, the 
chlorophyll content in some mutants increased significantly, but remained at a significantly lower level compared to WT. The simultaneous measurements of chlorophyll fluorescence and P700 absorbance indicated altered electron and proton transport, resulting to lower trans-thylakoid $\mathrm{pH}$-gradient, leading to lower NPQ in early growth phases.

\section{Conclusions}

As a result of insufficient regulation of linear electron transport, the acceptor side of photosystem I (PS I) was more reduced, creating conditions for enhanced oxidative damage due to reactive oxygen species. The shift of balance between PSII and PSI redox poises indicates lower PS I to PS II ratio in chlorina mutants compared to WT. Our results also suggest that chlorina mutant of wheat had lower capacity to increase the rate of cyclic electron flow around PSI, which makes these mutants more susceptible to environmental constraints. The severity of these effects varied in different mutants and growth phases. The relationships between leaf traits, photochemical responses and photosynthetic capacity were also examined.

\section{Acknowledgement}

This work was supported by the research project of the Scientific Grant Agency of Slovak Republic APVV-15-0721, VEGA-1-0831-17 and APVV-SK-CN-2015-0005).

\section{References}

Brestic, M., Zivcak, M., Hauptvogel, P., Misheva, S., Kocheva, K., Yang, X., Li, X., Allakhverdiev, S.I. (2018): Wheat plant selection for high yields entailed improvement of leaf anatomical and biochemical traits including tolerance to non-optimal temperature conditions. Photosynth Res. 136. 245-255. doi: 10.1007/ s11120-018-0486-z.

Driever, S.M., Lawson, T., Andralojc, P.J., Raines, C.A., Parry, M.A.J. (2014): Natural variation in photosynthetic capacity, growth, and yield in 64 field-grown wheat genotypes. J. Exp. Bot. 65. 4959-4973.

Klughammer, C., Schreiber, U. (1994): Saturation pulse method for assessment of energy conversion in PS. I. Planta 192. 261-268. doi:10.1016/j.cell.2015.03.019

Long, S.P., Marshall-Colon, A., Zhu, X.G. (2015): Meeting the global food demand of the future by engineering crop photosynthesis and yield potential. Cell 161. 56-66. doi:10.1093/jxb/eru253

Zhu, X.G., Long, S.P., Ort, D.R. (2010): Improving photosynthetic efficiency for greater yield. Annu Rev Plant Biol 61. 235-261. doi: 10.1146/annurev-arplant-042809-112206. 